\section{Gesundheitsförderung im Alter}

In mehreren Ländern existieren bereits präventive Programme, um ältere Menschen in ihrer Gesundheit zu unterstützen. Eine internationale Studie zeigt, dass mit einer persönlichen und mehrstufigen Beratung die Gesundheit älterer Menschen deutlich gesteigert werden kann. Der Schweizer Teil der Studie war über acht Jahre angelegt und entstand in Zusammenarbeit mit der Spitex. Mehr als 2000 über 65-jährige, selbständig zu Hause lebende Personen wurden in zwei Gruppen unterteilt: Die Vergleichsgruppe erhielt die übliche medizinische Betreuung, die Interventionsgruppe zusätzlich ein neues BeratungsAngebot. «Die Lebenserwartung bei Personen der Interventionsgruppe war höher als diejenige der Vergleichsgruppe», sagt Andreas Stuck von der Forschungsgruppe Geriatrie der Universität Bern und der Geriatrischen Universitätsklinik des Inselspitals.

(Universität Bern)

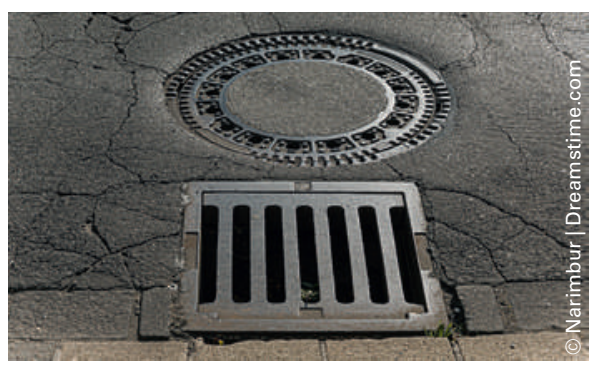

Pour mieux connaître la consommation de drogue des habitants, des chercheurs font parler les indices cachés dans les égouts.

Dans les entrailles de la ville Pour mieux connaitre la consommation de drogue des habitants, des chercheurs font parler les indices cachés dans les égouts. "On pourrait dire que la base de mon travail tient au fait que les consommateurs de drogues doivent eux aussi se rendre aux toilettes", déclare Ann-Kathrin McCall, ingénieur en sciences de l'environnement. Leur urine évacue des résidus et des métabolites d'amphétamines, d'ecstasy et de cocaïne. Il suffit de procéder à une analyse chimique pour savoir ce qui se consomme dans une ville. Le problème est qu'entre les toilettes et les stations d'épuration, les drogues et leurs métabolites sont modifiés par des micro-organismes et par d'autres processus. Grâce à sa recherche, AnnKathrin McCall tente de découvrir ce qui se passe exactement pendant cette phase pour pouvoir analyser de manière encore plus fiable.

(Fonds national suisse de la recherche scientifique, FNS)

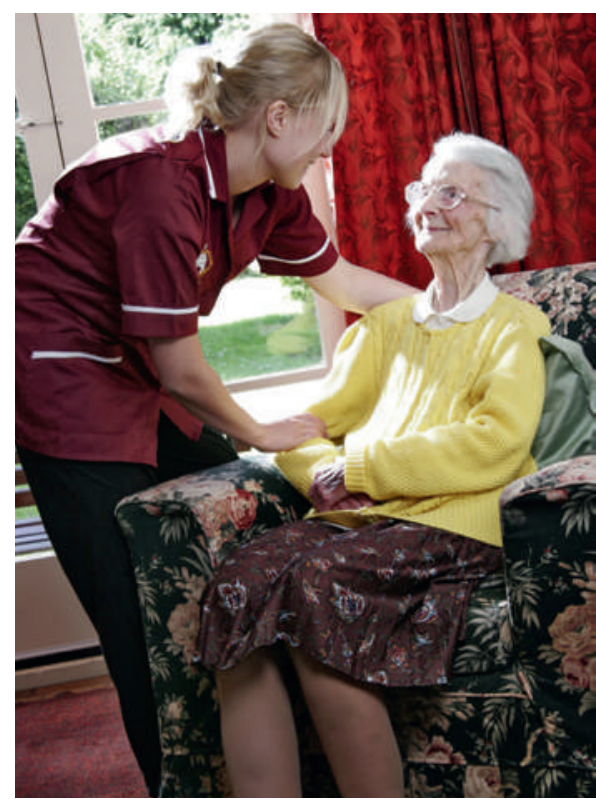

Eine internationale Studie zeigt: Ältere Menschen, die auf sich achtgeben, leben länger.

\section{Sucht als Familiengeheimnis}

Wenn Papi oder Mami trinkt: Für rund 100000 Kinder in der Schweiz ist dies Realität. Überforderung, Scham- und Schuldgefühle prägen den Alltag der Kinder wie auch der Eltern. Sucht Schweiz stellt zwei neue Angebote vor, welche eine wichtige Lücke in der Unterstützung suchtbelasteter Familien schliessen. Die Website www.elternundsucht.ch zeigt beiden Elternteilen sowie weiteren Bezugspersonen betroffener Kinder, was sie für das Kind tun können, wie einem Kind die Suchterkrankung erklärt und wie Stabilität erlangt werden kann. Das Bilderbuch mit dem kleinen Hund Boby und seinem alkoholkranken Herrchen richtet sich an Suchtfachleute und Lehrpersonen, damit sie mit Kindern über die Alkoholkrankheit des Vaters oder der Mutter sprechen können. Das Buch wurde neu um vier Hörgeschichten mit Themen aus dem Alltag der Kinder erweitert.

(Sucht Schweiz)

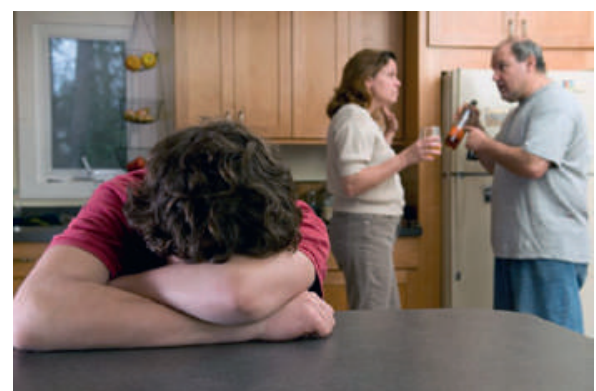

Überforderung, Scham und Schuldgefühle prägen den Alltag von Kindern mit suchtkranken Eltern. Sucht Schweiz stellt zwei neue Angebote zur Unterstützung vor. nutrinfo ${ }^{\circledR}$ - Le service d'information

nutritionnelle

nutrinfo ${ }^{\circledR}$, une offre de la Société Suisse de Nutrition, répond aux questions sur le thème de l'alimentation. nutrinfo ${ }^{\circledR}$ n'obéit à aucun intérêt commercial. Le service a été mis en place à la demande de l'Office fédéral de la sécurité alimentaire et des affaires vétérinaires (OSAV). nutrinfo ${ }^{\circledR}$ offre des feuilles d'informations, des brochures et des supports d'enseignement. Le service procure des adresses, propose des conférenciers, des formateurs, une documentation spécialisée et une médiathèque sur l'alimentation. Les consultations sont généralement gratuites.

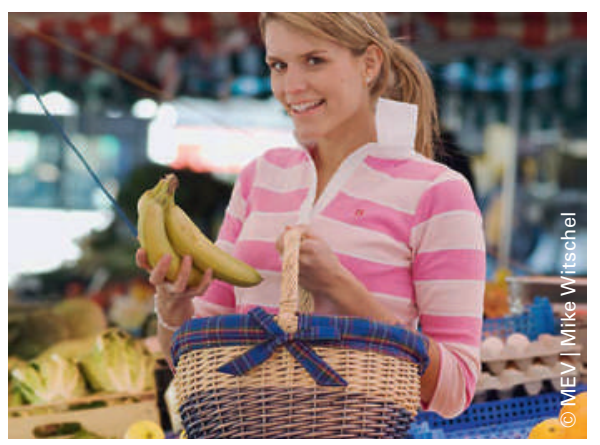

nutrinfo ${ }^{\circledR}$, une offre de la Société Suisse de Nutrition, répond aux questions sur le thème de l'alimentation.

Beobachter-Ratgeber "Wenn die Psyche streikt" Der neue Beobachter-Ratgeber "Wenn die Psyche streikt", herausgegeben von der Stiftung Pro Mente Sana, zeigt konkret, wie Mitarbeitende, Führungspersonen und Betriebe die psychische Gesundheit im Arbeitsumfeld fördern können. Er liefert zudem klare Argumente, warum es sich lohnt, in die psychische Gesundheit am Arbeitsplatz zu investieren. Nach dem erfolgreichen Ratgeber "Ganz normal anders" hat der Stiftungspräsident von Pro Mente Sana und Chefarzt der Spitäler fmi Interlaken, Dr. Thomas Ihde-Scholl, einen weiteren praktischen Ratgeber für die Praxis geschrieben.

(Pro Mente Sana)

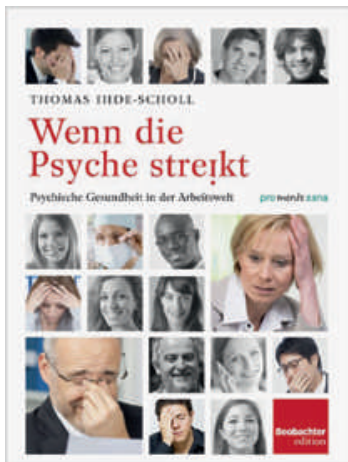

Zeigt, wie die psychische Gesundheit am Arbeitsplatz gefördert werden kann: der neue Beobachter-Ratgeber "Wenn die Psyche streikt». 